\title{
Analysis of Photo Neutron Dose to Patient from a 15 MV Linear Accelerator
}

\author{
Rajesh.K.R, R.Ganapathi Raman*
}

\begin{abstract}
Medical Linear Accelerator produces high-intensity bremsstrahlung beam, with typical energy ranges from 4 to $18 \mathrm{MV}$ for treatment purposes. The materials including patient exposed to this high energy spectrum lead to a photonuclear reaction in them. In these Photon energy domains, photo neutron production ( $n, p$, and $\gamma, n)$ is the major reaction channel. This is important as Carbon, Oxygen, Hydrogen, and Nitrogen are the predominant elements in a typical human being and having low photon neutron threshold. Apart from this, the equipment involved in a radiation treatment including Head, Couch, immobilization accessories, etc contributes to photo neutron production. The present study was recorded and analyzed the photo neutron spectra due to $15 \mathrm{MV}$ medical linear accelerator using CR-39 Solid State Nuclear Track Detector and image processing software.
\end{abstract}

Index Terms: Solid State Nuclear Track Detector (SSNTD), CR-39(Columbia Resin \#39), Chemical Etching, EXFOR, Karma calculation

\section{INTRODUCTION}

Radiation treatment is a branch of medicine in which ionizing radiations are used to treat harmful malignant diseases. Radiotherapy may be curative in a type of malignant growth if they're localized to at least one space of the body[1]. It might likewise be used as palliative treatment. Neutrons are produced as a result of photonuclear reactions within the target, field-flattening filters and beam collimators in linear accelerators operating at high energies[2]. With the target of investigating photo neutron dose from a linac generally used for treatment purpose, we have got recorded photo-neutron spectra using $15 \mathrm{MV}$ medical linear accelerator[3]. The neutrons thus produced are recorded in a CR39 Solid State Nuclear Track Detector (SSNTD) and examined using image processing software system and investigated for absorbed

Revised Manuscript Received on July 05, 2019.

Rajesh.K.R, Department of Physics, Noorul Islam Centre for Higher Education, Kumaracoil, Kanya kumari District. Tamilnadu, India.

R.Ganapathi Raman, Department of Physics, Noorul Islam Centre for Higher Education, Kumaracoil, Kanya kumari District. Tamilnadu, India. ganapathiraman83@gmail.com dose basically from hydrogen gas, Carbon, oxygen and nitrogen interactions as these are the antiquated parts within the typical human tissue [4]. A Neptun 10PC medical linear accelerator, with the Mont-Carlo code MCNPX emitted the photo neutron contamination. That photo neutron contamination measured and conferred[5]. Conferred with photo neutron within the vicinity of Siemens Primus linear accelerator. This work incorporates the four elements of measurements. (Portable neutron rem-meter calibrated with shadow cone methodology was used for environmental survey within the vicinity of treatment room neutron detection module, as well as the BF3 proportional counter tube Neutron activation analyses of $\mathrm{Au}$ foil [6,7]. Powdered $\mathrm{P} 2 \mathrm{O} 5$ was used to measure the thermal and quick neutron fluence within the x-ray field. Quick neutron dosimetry with CR-39 Plastic Plate by and reported the application of CR-39 in neutron dosimetry.

\section{MATERIALS AND METHODS}

\section{Medical linear Accelerator}

The linac is used to accelerate charged particles from High frequency (HF) electromagnetic waves. It resembles electrons to high energies through a linear tube. The high-energy electron beam is likewise utilized for treating shallow malignant growths (superficial cancer), or it tends to be made to strike an objective to create $\mathrm{x}$-ray beams for treating deep-seated tumors. The medical linear accelerator can accelerate the electron from kinetic energy (K.E) starting from 4 - $25 \mathrm{MeV}$.

\section{Neutron}

The neutron is one of the fundamental particles. It has no net electric charge and a mass marginally bigger than a proton. The free neutron has contained a mass of about $1.675 \times 10-27$ $\mathrm{kg}$. It is equivalent to $939.6 \mathrm{MeV} / \mathrm{c} 2$, or $1.0087 \mathrm{u}$. The neutron contains an average mean square radius of about $0.8 \times 10-15$ $\mathrm{m}$, and it's a spin- $-1 / 2$ fermion and a mean lifetime of 15 minute Classification of neutrons by energy like, Thermal: $\mathrm{E}<1$ electron volt $(0.025 \mathrm{eV})$, Epithermal: 1 electron volt $<\mathrm{E}<10$ $\mathrm{keV}$, Fast: $>10 \mathrm{keV}$ and Energy Deposition by Neutrons. Like photons, neutrons are uncharged and do not act with orbital electrons. Neutrons can able to travel significant distances through matter while not interacting. Neutrons can interact with atomic nuclei through numerous mechanisms. That is 1) Inelastic scattering 2) Elastic scattering 3) neutron 


\section{Analysis of Photo Neutron Dose to Patient from a 15 MV Linear Accelerator}

capture 4) Nonelastic scattering 5) nuclear reaction. The kind of interaction depends on the neutron energy.

\section{CR-39 (Columbia Resin \#39)}

CR-39 is a polymeric compound and solid-state nuclear track detector that is highly applied for neutron dosimetry. CR-39 detects neutrons by the harm trails from the nuclei of its constituent atoms that are carbon, hydrogen, oxygen. The use of a hydrogenous radiator related with CR-39 can ready to improve its reaction, because of the additional proton recoils produced among the radiator. Once combined with radiators, CR-39 can find neutrons over reasonably wide energy range differ from around $100-20 \mathrm{MeV}$. The energy range, dose equivalent response (cmv2 $\mathrm{mSv}-\mathrm{I}$ ), and furthermore the lower limit of detection is dependent on the sort of CR-39 material, a thickness of the radiator and the particular etching process used.

\section{Chemical Etching}

Etching is the actual immersion of the part into the chemical bath, and furthermore chemical action on the part to proceed milled Chemical edge is that the subtractive producing method of using baths of temperature regulated etching chemicals compounds to remove the material to make an object with the required form of the conventional Etchants are Keller's reagent and $\mathrm{NaOH}$. Energetic (vigorous) particles colliding with the polymer structure leave a path of broken chemical bonds inside the CR-39. Once immersed in a focused alkali solution hydroxide ions attack and break the polymer structure, etching away the majority of the plastic at a nominally settled rate.

\section{Python}

Python is widely used in the world as interpreted, general-purpose, highly effective programming language. Its design philosophy emphasizes code readability, and its syntax grants programming developers to explicit their specific ideas in fewer lines of code than achievable in programming languages like java. Memory consumption was not much worse than $\mathrm{C}++$ or $\mathrm{C}$ and sometimes better than Java. Within this study, Python compiler is used for resolving neutron energy information from chemically etched CR-39 foil microscopic image that is used to calculate neutron dose.

\section{EXFOR}

EXFOR is the library for collection, exchange, recovery, storage of experimental nuclear reaction information database. The library is the result of a worldwide co-operation, specifically the global Network of nuclear reaction data Centre's (NRDC) that is regulated by the iaea nuclear information Section (NDS). At present (May 2008), the EXFOR database contains about 17,000 works with around 129,000 data information tables, representing as, A) A "complete" compilation of low-energy experimental neutron-induced reaction data B) A specific compilation of photon-induced, heavy-ion-induced, and high energy neutron-induced reaction data, C) A less complete compilation of charged-particle-induced reaction data.

The ICRU composition for muscle has been assumed in most cases for neutron-dose calculations, lumping the $1.1 \%$ of "other" minor parts together with oxygen to create a simple and basic four-elemental (Hydrogen, Oxygen, Carbon, Nitrogen) composition.

\section{Elements of Human Body}

The ICRU composition for muscle has been assumed in most cases for neutron-dose calculations, lumping the $1.1 \%$ of "other" minor parts together with oxygen to create a simple and basic four-elemental (Hydrogen, Oxygen, Carbon, Nitrogen) composition. The amount of each and every chemical compound substances found in the human physical body, from most to least abundant is given in Initial data from in that table was created from John, Emsley, the elements, 3rd ed., Oxford, Clarendon Press, 1998. For every element, there's the amount in mass units in an average in 70-kilogram for every individual, the amount of the element, and the length of the facet of a cube that might contain the pure element. The body's 43 kilograms of oxygen is found fundamentally as a part of the water that makes up 70th of overall human body weight. Rubidium is one of the most abundant elements in the human body that is in $0.68 \mathrm{~g}$. it does not have any important biological role (silicon that is slightly high, simple, might have a metabolic function). Vanadium is the body's another most abundant element it is in $0.11 \mathrm{mg}$. It has an identified biological role, take the place of cobalt in $3 \mathrm{mg}$ and the latter being a constituent of vitamin b12.

\section{Photo Neutron Measurements}

Neutrons are non-ionizing fundamental particles and it is known by detection of ionized particles emitted in neutron interaction. The foremost common reactions are $(n, p),(n, \alpha)$, $(\mathrm{n}, \gamma)$, and proton recoil. The dosimeters are often scintillators, solid-state, track detectors, photographic film, TLD and different types. The energy which is Higher than the $10 \mathrm{keV}$ dose in the human physical body is dominated by the contribution of recoil protons come from an elastic scattering of hydrogen nuclei. Below that energy dose important due to y-rays come from thermal-neutron capture in hydrogen. So the neutron quality issue increases slightly higher than 10 $\mathrm{keV}$.

\section{KERMA CALCULATION AND MEASUREMENT}

Neutron dose is calculated using the kerma. The kerma factor is for neutrons of energy, for a given material is defined as the ratio of the neutron kerma $\mathrm{K}$ and the neutron fluence. For neutron energy lesser than $20 \mathrm{MeV}$, the information data can be taken from the ENDF/B-V data file. For higher energies, the data information is from relied-on calculations only. Higher than $15 \mathrm{MeV}$ the amount of nuclear reactions increases and well contributes to the kerma, with strong uncertainty within the 
cross-section. For single neutron energy, type of target atom and sort of interaction, the kerma that results from a neutron fluence $\varphi\left(\mathrm{cm}^{-}\right)$at a point in aMedium is given by

$$
\mathbf{K}=1.602 \times \mathrm{lo}^{\wedge}(-8) *(\varphi) *(\sigma) *\left(\mathbf{N}_{\mathrm{T}}\right) * \mathrm{~m}^{\wedge}(-1) *\left(\mathbf{E}_{\mathrm{tr}}\right) \mathrm{Gy}
$$

Where, Nis the number of target atoms in the irradiated sample, $\mathbf{m}$ is the sample mass in grams, ois the interaction cross section in $\mathrm{cm}^{\prime}$ / (target atom) and $\mathbf{E}_{\mathrm{tr}}$ is the total kinetic energy $(\mathrm{MeV})$ given to charged particles per interaction, $\mathrm{K}$ is given in rad or centi grays, and its value is equal to the absorbed dose $\mathrm{D}$ at the same point under the typical CPE conditions In our procedure we have averaged the unit volume throughout the body and taken as per kilogram so the averaging factor taken in above equation becomes reduced to $\mathbf{K}=(\varphi) *(\sigma) *\left(\mathbf{N}_{\mathrm{T}}\right) *\left(\mathbf{E}_{\mathrm{tr}}\right) \mathbf{G y}$

As a result of the short range of the heavy charged secondary particle is produced in photon interaction. CPE is normally well approximated and thus absorbed dose can be thought to be equal to KERMA at any point of interaction up to $20 \mathrm{MeV}$.

\section{APPLICATION OF CR39 SSNTD IN NEUTRON SPECTROSCOPY}

A CR-39 SSNTD is a hydrocarbon polymer film used to record proton recoil tracks with the elastic scattering of hydrogen gas nucleus. Recoil protons create a latent track on the SSNTD film by scission on the poly-carbon chain by the energy deposition of the recoiled proton. Length of latent track is adequate the range of proton recoiled within the CR-39 material. With calibration of the diameter of etched track versus energy, the energy of the recoiled proton is measured. From the angle of recoil $\Theta$, incident neutron energy can be calculated as.

$$
\mathbf{E p}=\mathbf{E}_{\mathbf{n}} \cos ^{2} \Theta
$$

Where, Ep, En, $\Theta$ and is the energy of recoiled proton, incident neutron energy and the is calculated from major and $\square$ angle of recoil respectively. Recoil angle $\Theta$ minor axes of the track ellipse.

\section{Experimental Setup and Data Recording}

A piece $300 \mu \mathrm{m}$ thick CR39 Solid State Track Detector (SSNTD), having $1 \mathrm{~cm} 2$ area and physical density $1.3 \mathrm{~g} / \mathrm{cm} 3$ manufactured by Pershore Mouldings limited tm, UK, has been used for the measurement. SSNTD was placed on the poly-carbon treatment couch for a planned four field Box technique for a ca cervix patient. Exposed SSNTD was etched in $\mathrm{NaOH}$ chemical with 600C, standard etching conditions of $6 \mathrm{~N}$, and $6 \mathrm{Hrs}$ with constant stirring. The etched film was observed by an optical microscope of $40 \mathrm{X}$ magnification and the image view was snapped by an $8 \mathrm{Mega-Pixel} \mathrm{camera.} \mathrm{The}$ microscope image of CR39 is investigated with Image software system. From the image, Two-dimensional profiles of the area containing tracks were generated. The every track profiles, in this area, were fitted with ellipse equation using Gnuplot, the important diameter, minor diameter and angle of recoil was estimated from fitted parameters. The energy of the recoiled protons has been estimated using track diameter - energy calibration for protons in standard etching conditions $(6 \mathrm{~N} \mathrm{NaoH}, 600 \mathrm{C}$, and 6Hrs) and the energy of incident neutrons was deducted using eqn. (1). A typical track fits for single track and multiple tracks are shown in. The difference observed is well within the statistical fluctuation less than 4 . The data information the info the information were binned with a width of $250 \mathrm{KeV}$ and intrinsic corrections are applied by accounting elastic scattering cross section data were collected from EXFOR-ENDF (Evaluated Nuclear section (data File). the midpoint of each and every bin is taken as the average A number of scattering centers per unit area were calculated from energy (E). The physical density and molecular composition. The image of the linac is given in

\section{RESULTS AND DISCUSSION}

\begin{tabular}{|c|c|c|}
\hline Volume of purified element & $\begin{array}{c}\text { Mass of element in a } 70 \text { - } \\
\text { kg person }\end{array}$ & Main Elements \\
\hline $37 \mathrm{~L}$ & $43 \mathrm{~kg}$ & Oxygen \\
\hline $7.08 \mathrm{~L}$ & $16 \mathrm{~kg}$ & Carbon \\
\hline $98.6 \mathrm{~L}$ & $7 \mathrm{~kg}$ & Hydrogen \\
\hline $2.05 \mathrm{~L}$ & $1.8 \mathrm{~kg}$ & Nitrogen \\
\hline $645 \mathrm{~mL}$ & $1.0 \mathrm{~kg}$ & Calcium \\
\hline $429 \mathrm{~mL}$ & $780 \mathrm{~g}$ & Phosphonss \\
\hline $162 \mathrm{~mL}$ & $140 \mathrm{~g}$ & Potassium \\
\hline $67.6 \mathrm{~mL}$ & $140 \mathrm{~g}$ & Sulfur \\
\hline $103 \mathrm{~mL}$ & $100 \mathrm{~g}$ & Sodium \\
\hline $63 \mathrm{~mL}$ & $95 \mathrm{~g}$ & Chlorine \\
\hline $10.9 \mathrm{~mL}$ & $19 \mathrm{~g}$ & Magnesium \\
\hline $0.53 \mathrm{~mL}$ & $4.2 \mathrm{~g}$ & Iron \\
\hline $1.72 \mathrm{~mL}$ & $2.6 \mathrm{~g}$ & Fluorine \\
\hline $0.32 \mathrm{~mL}$ & $2.3 \mathrm{~g}$ & Zinc \\
\hline $0.43 \mathrm{~mL}$ & $1.0 \mathrm{~g}$ & Silicon \\
\hline $22 \mu \mathrm{L}$ & $60 \mathrm{mg}$ & Aluminum \\
\hline $4.06 \mu \mathrm{L}$ & $20 \mathrm{mg}$ & Iodine \\
\hline $5.3 \mathrm{~nL}$ & $0.1 \mathrm{mg}$ & Uranium \\
\hline $10 \mathrm{~nL}$ & $0.2 \mathrm{mg}$ & Gold \\
\hline $18 \mathrm{~nL}$ & $0.11 \mathrm{mg}$ & Vanadium \\
\hline
\end{tabular}

Table 1. Main elements of human body

\section{Variables and calculations}

Patient Selection, 57 Years old female with Ca cervix, Weight: $69 \mathrm{Kg}$, Height: $167.6 \mathrm{~cm}$. Thickness of $24.5 \mathrm{~cm}$ at Isocenter. Four field Box Technique with 15MV Photon used with maximum field size of $20 * 22 \mathrm{~cm} * \mathrm{~cm}$ with MLC shaped (Planned from TPS)

$$
\operatorname{Kerma}=(\varphi) *(\sigma) *\left(N_{T}\right) *\left(E_{t r}\right) G y
$$

Where $\varphi$ - Fluence in nuber

$\sigma$ - Cross-section in barn (convert to $\mathrm{m}^{\wedge} 2$ )

$\mathrm{N}_{\mathrm{T}}$ - Number of atom $/ \mathrm{Kg}$

$\mathrm{E}_{\mathrm{tr}}-$ Energy transfer to neutron in Mev (convert to J) 


\section{Analysis of Photo Neutron Dose to Patient from a 15 MV Linear Accelerator}

1 barn $-10^{\wedge}(-28) \mathrm{m}^{\wedge} 2$

$1 \mathrm{eV}-1.602 * 10^{\wedge}(-19) \mathrm{J}$

$1 \mathrm{~Gy}=1 \mathrm{~J} / \mathrm{Kg}$

$1 \mathrm{~Gy}=100 \mathrm{cGy}$

Fluence $\phi=$ count $* 4 * \mathrm{pi} * \mathrm{r}^{\wedge} 2 / 0.035\left(\mathrm{~cm}^{\wedge} 2 / \mathrm{cm}^{\wedge} 2\right)$

Where $\mathrm{r}$ - half thickness of Patient $(12.25 \mathrm{~cm})$

Number of atom $\mathrm{N}_{\mathrm{T}}=$ abundance\%*weight $* \mathrm{~N}_{\mathrm{A}}$ /(mass numban)d $7.3 \mathrm{MeV}$ respectively. Thus the patient will be exposed to $\mathrm{N}_{\mathrm{A}}-6.023 * 10^{\wedge} 23$

Table 2. The Kerma factor calculated for Neutron dose

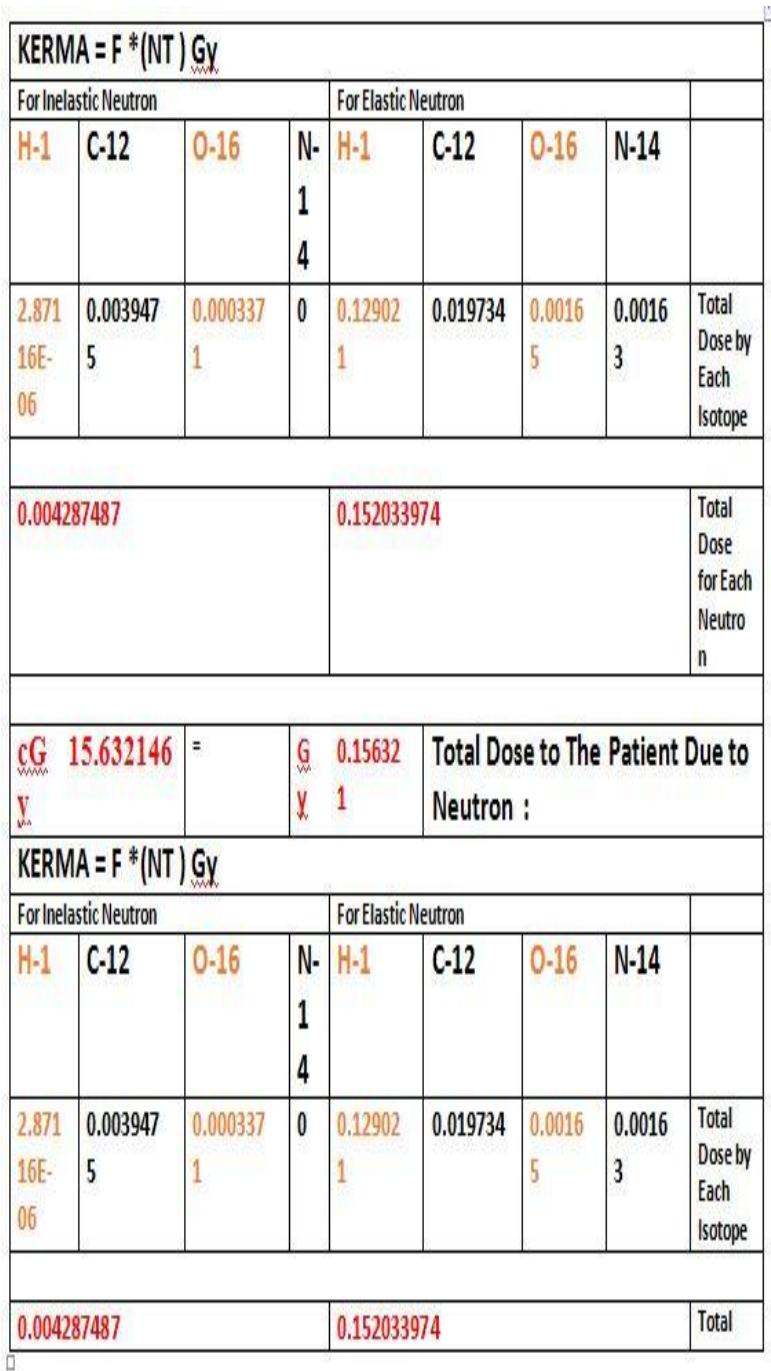

From the above observations, it is clearly seen that significant photo-neutrons of varying energy are produced from a variable component of the LINAC treatment head. Further, the beam is used for treating tumors inside the body which consists of, primarily, Carbon, Hydrogen, and Oxygen along with Nitrogen, Calcium, and Phosphorus. Similar photo-neutron production is expected from the couch, Immobilizer and other patient supporting accessories in which Carbon, Hydrogen, and Oxygen are the basic ingredients (Table.3). Hence they cause photo-neutron production even at low photon energies say 4 and $6 \mathrm{MVs}$ which are commonly used. Further $15 \mathrm{MV}$ lead to photoneutron production from many of the elements including Tungsten, Lead, Tantalum Nitrogen, Phosphorus and so on. Nitrogen and Phosphorus have a lesser threshold for photo-proton emission with thresholds at $7.55 \mathrm{MeV}$ external neutrons from LINAC head and neutrons and protons generated within the body. This is added with the contributions from patient supporting accessories. Further fast neutron may induce secondary neutron emission within the body.

Neutrons produced in the tumor also will interact with peripheral tissues. All these neutrons get thermalized within the body, making the neutron more lethargic. All the above dose may cause radiation-induced second malignancies and post-irradiation second malignancies rather than recurring cancers. Because of the lethargic behavior of energy deposition, neutron transport inside the body will take a larger peripheral dose which should be analyzed well for resolving the problem of secondary toxicities. Also dose to neutron sensitive organs such as the spine, parotid etc. are also be analyzed in a similar manner. As far as the reports on therapies are concerned it is seen that this heavy overdose is completely ignored or neglected. In fact, these excess doses of photo-neutrons are to be account properly and necessary corrections should be incorporated in the treatment planning. Otherwise, this will lead to post-treatment second malignancies. Protocols, limits, and guidelines are also should be modified accordingly.

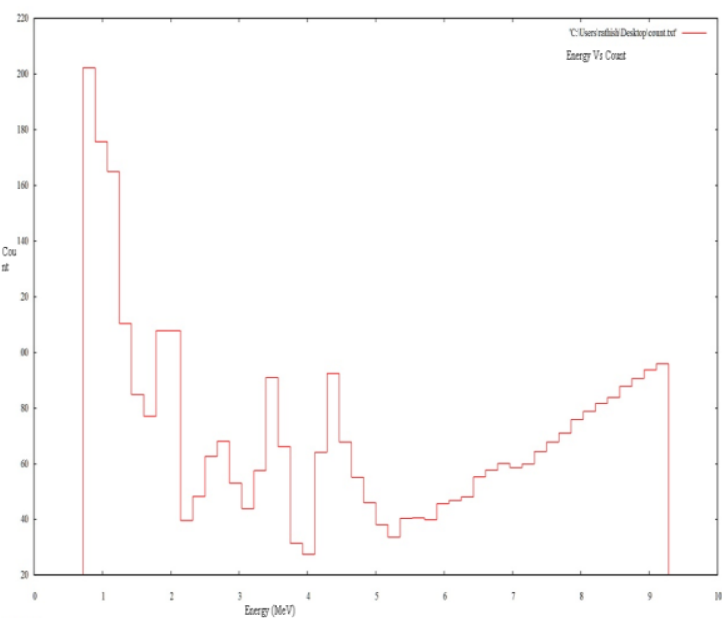

Figure1. Represent the Neutron Spectrum 


\begin{tabular}{|c|c|c|c|c|c|}
\hline $\begin{array}{c}\text { \% in } \\
\text { Human } \\
\text { body }\end{array}$ & $\begin{array}{c}\text { \% } \\
\text { Abundanc } \\
\mathrm{e}\end{array}$ & $\begin{array}{c}\text { Mas } \\
\text { s of } \\
\text { Ato } \\
\mathrm{m}\end{array}$ & $\begin{array}{c}\text { Symbo } \\
1\end{array}$ & Name & $\mathrm{Z}$ \\
\hline 9.5 & 99.9885 & 1.007825 & $\begin{array}{c}1 \\
\mathrm{H}\end{array}$ & $\begin{array}{l}\text { Hydroge } \\
\mathrm{n}\end{array}$ & 1 \\
\hline 18.5 & 98.93 & $\begin{array}{c}12.00000 \\
0\end{array}$ & $\begin{array}{c}2 \\
\mathrm{C}\end{array}$ & Carbon & 6 \\
\hline 3.2 & 99.632 & $\begin{array}{c}14.00307 \\
4\end{array}$ & $\begin{array}{c}4 \\
\mathrm{~N}\end{array}$ & Nitrogen & 7 \\
\hline 65 & 99.757 & $\begin{array}{c}15.99491 \\
5\end{array}$ & $\begin{array}{c}16 \\
0\end{array}$ & Oxygen & 8 \\
\hline
\end{tabular}

Table 3. Mass of Elements of human body

\section{CONCLUSION}

15 MV Medical Linear Accelerator n) reactions occur $\square$ shows a significant amount of neutrons production from $(\gamma$ at various parts of the machine, the continuous behavior of the photon spectrum is one of the major factors for high neutron production. Similar photo-neutron production is expected from the couch, Immobilizer and other patient supporting accessories in which Carbon, Hydrogen, and Oxygen are the basic ingredients. The human body is consisting primarily of Carbon, Hydrogen, and Oxygen along with Nitrogen, Phosphorus and Calcium, which are having a photoneutron threshold at low energy. Thus the patient will be exposed to external neutrons from LINAC head and neutrons and protons generated within the body. This is added with the contributions from patient supporting accessories. Further fast neutron may induce secondary neutron emission within the body. Neutrons produced in the tumor also will interact with peripheral tissues. All these neutrons get thermalized within the body, making the neutron more lethargic. The entire above dose may cause radiation-induced second malignancies and also the dose to neutron sensitive organ such as the spine, parotid should be monitored. The probability of this excess dose of photoneutron should be explored to incorporate in the treatment planning to help to achieve the goal of Radiotherapy and Radiation Protection.

\section{REFERENCES}

1. Castillo F, Espinosa G, Golzarri JI, Osorio D, Rangel J, Reyes PG, Herrera JJ. Fast neutron dosimetry using CR-39 track detectors with polyethylene as radiator. Radiation Measurements. 2013 Mar 1;50:71-3.

2. Zabihinpoor S, Hasheminia M. Calculation of neutron contamination from medical linear accelerator in treatment room. Adv Studies Theor Phys. 2011;5(9):421-8.

3. Lin JP, Chu TC, Lin SY, Liu MT. The measurement of photoneutrons in the vicinity of a Siemens Primus linear accelerator. Applied Radiation and Isotopes. 2001 Sep 1;55(3):315-21.
4. Wu RK, McGinley PH. Neutron and capture gamma along the mazes of linear accelerator vaults. Journal of applied clinical medical physics. 2003 Mar;4(2):162-71.

5. Cheng L, Brzozowska B, Sollazzo A, Lundholm L, Lisowska H, Haghdoost S, Wojcik A. Simultaneous induction of dispersed and clustered DNA lesions compromises DNA damage response in human peripheral blood lymphocytes. PloS one. 2018 Oct 31;13(10):e0204068.

6. Griffin PJ, Sjöstrand H, Simakov S. Nuclear Reaction Data and Uncertainties for Radiation Damage: Summary Report of the Technical Meeting, IAEA Headquarters, Vienna, Austria13-16 June 2016.

7. Cyriac TS, Musthafa MM, Raman RG, Haneefa KA, Bhasi S. Out-of-field photon dosimetry study between 3-D conformal and intensity modulated radiation therapy in the management of prostate cancer. International Journal of Radiation Research. 2015 Apr 1;13(2):127-34.

Unlike surgery and chemotherapy, most radiation treatments are completely non-invasive. Patients that cannot go through the medical surgery or chemotherapy in view of their medicinal condition typically receive radiotherapy. In radiotherapy, ionizing radiations are used to destroy the tumor cells while minimally influencing the surrounding ordinary tissue [1]. While radiation goes through the body, it will bind with the healthy tissue in addition to the tumor is a non-avoidable issue. The uncontrolled growth of tumors suggests that the majority of its cells lie in the mitotic (M) phase of the cell cycle. This turns out to be maximum radiosensitive [2].

Taking advantage of the growth properties of tumors, two different quantities of radiation therapy is established. First is Tumor Control Probability (TCP) and other is Normal Tissue Complication Probability (NTCP). The therapeutic ratio is the proportion of these two quantities for a specific radiation dose which relates the tumor response to a specific measure of surrounding ordinary tissue damage. There are two common radiation delivery techniques in radiotherapy: brachytherapy and external beam radiotherapy [3]. Brachytherapy is the administration of radiation by temporarily implanting a source into the tumor allowing greater sparing of healthy tissue and implanting permanently possible. Ultimately, the choice of treatment depends on the location, size, and type of tumor as well as patient health [4]. External beam radiotherapy is defined as radiation delivery from a source that is exterior to the body and is the most common technique that accounts for $80 \%$ of all radiation treatments. Depending on the tumor, different types of external beam radiotherapies are used for treatments.

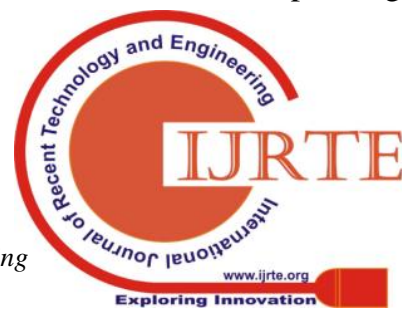




\section{Analysis of Photo Neutron Dose to Patient from a 15 MV Linear Accelerator}

Conventional 3D conformal involves the intersection of one or more conformal-shaped beams at the tumor and IMRT (Intensity Modulated Radiation Therapy) applies many intensity-modulated beams using inverse treatment planning among the various external beam modalities [5]. For superficial tumors, orthovoltage $\mathrm{x}$-ray units, with shallow depth dose curves deliver radiation with energies ranging from $10 \mathrm{KeV}$ to $500 \mathrm{KeV}$. Conversely, for deep-seated tumors, linear accelerators deliver high-energy radiation ranging from $2 \mathrm{MV}$ to $25 \mathrm{MV}$. Inevitably, while gaining the ability to reach deeper into the body, more healthy tissues are also irradiated.

Fluence explained equivalent dose and sources of photo neutrons measured in a medical accelerator [6]. The medical linear accelerator measurement of Neptun 10PC with the Mont-Carlo code emits MCNPX, the photo neutron contamination. In 1988, the American Association of Physicists in Medicine (AAPM) published report 19 (TG 27) on neutron measurement around the X-ray radiotherapy high energy machines. These gave the recommendation for methods and instrumentation for neutron measurements inside and outside the linac treatment room and provide a detailed step-by-step description of these methods. Increased use of medical linac and complex treatment techniques in cancer created the need to verify the old calculation results with updated photonuclear cross section data and new Monte Carlo methods. Fast CR-39 Plastic Plate neutron dosimetry revealed the application of CR-39 in neutron dosimetry. The photo neutron dose equivalent to patients in a Siemens KD-S radiotherapy accelerator operating at $18 \mathrm{MV}$ was done through measurements and Monte Carlo simulations [7].

Neutron production because of photonuclear interactions in linear accelerators has been an unwanted by-product of radiotherapy treatments. Interaction of photons with materials or air produce secondary neutrons called contaminated neutrons. Compared to photons, neutrons can produce carcinogenic mutations up to twenty times more effectively and contribute additional dose to the patient during treatment. The Department of Radiation Oncology, Caritas Cancer Institute is equipped with Siemens Primus Plus linear accelerator. It has photon energies of $15 \mathrm{MV}$ and $6 \mathrm{MV}$ and electron energies of $18 \mathrm{MeV}, 15 \mathrm{MeV}, 12 \mathrm{MeV}, 9 \mathrm{MeV}$ and $6 \mathrm{MeV}$. The maximum dose rate is $600 \mathrm{MU} / \mathrm{min}$. The Multi-leaf collimator system is fifty-eight pairs leaf MLC that gives leaf resolution of $1 \mathrm{~cm}$ at isocenter for $20 \mathrm{~cm}$ central of $40 \mathrm{~cm} \times 40 \mathrm{~cm}$ field. In this present study neutron, CR39 solid-state nuclear track detector is used to perform the detection and Geant 4 application is used to access the dose. Even though the neutron dose is less, considering its biological effects with respect to the photon dose, it has to be accounted while in the radiotherapy planning.

\section{MATERIALS AND METHODS}

The linac is used to accelerate charged particles from High frequency (HF) electromagnetic waves. The electrons are stimulated to high energies through a linear tube. These high-energy electron beams similarly treats shallow malignant growths. It tends to strike an objective to create $\mathrm{x}$-ray beams for deep-seated tumor treatments. Hydrogen thyratrons are the direct current power supply for the modulator that has a pulse-forming network with switch tube. This results in a flat-topped high voltage pulse from the modulator area to DC pulse for a few microseconds of the period. At the same time, these pulses dispatched to a klystron or magnetron and then to the electron gun. Pulsed microwaves are created in the klystron or magnetron. It is injected towards the accelerator tube structure through a wave-guide system. The electron gun produces the proper instant electrons, which is the pulse injected into accelerator wave-guide or accelerator structure that is to be continued in a copper tube with its interior detached. In higher energy linacs, the structure of an accelerator is exceedingly long as it is positioned horizontally. The electrons were bent by means of an appropriate angle commonly around 270 or 90 degrees between the target and accelerator. The beam transport system is carried out by the way of precision bending of an electron beam. It consists of focusing coils, focusing coils and other different components.

When photons collide with a nucleus produces Photo neutrons. Figure 1 shows the setup of photo neuron production. A therapeutic photon beam composed of medical linear accelerators is used in external beam radiotherapy. An accelerating mono-energetic electron generated focuses the above-mentioned beams towards a target. The interaction of electrons with the target material causes them to decelerate and produce bremsstrahlung photons. This results in a beam of photons that can be focused on a tumor using various collimators.

At photon energies above $7 \mathrm{MV}$, neutrons can be produced through interactions with materials in the linear accelerator in a process known as photo neutron production. This process can only happen when the incident photon energy is more compared to the threshold energy of $(\gamma, n)$ reaction. In table 1 , the thresholds are unique to each element and are calculated from the Q-value of the photonuclear reaction. Photo neutron production is a specific interaction of the more general photodisintegration. Photodisintegration is the interaction between a high energy photon and the nucleus of an absorber which can lead to the production of neutrons $(\gamma, n),(\gamma, 2 n)$, protons $(\gamma, p)$, alpha particles $(\gamma, \alpha)$ and a combination of $(\gamma, n p)$ and $(\gamma, n \alpha)$. 


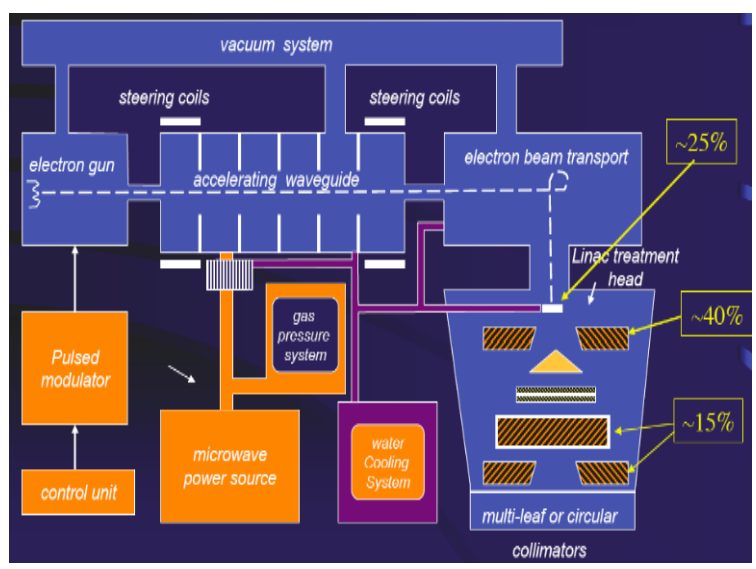

Fig.1 Setup of photo neuron production

Table 1 Threshold values of elements

\begin{tabular}{|l|l|}
\hline Threshold $[\mathrm{MeV}]$ & Element \\
\hline 1847.41 & Tungsten \\
\hline 6310.85 & Copper \\
\hline 5611.2 & Iron \\
\hline 1817.58 & Tantalum \\
\hline 2076.74 & Lead \\
\hline
\end{tabular}

CR-39 is a recently discovered versatile plastic track detector. The CR-39 detectors can be located very close to a source without being damaged. Like a photographic realistic film, CR-39 is a model of an integrating detector. In the present work, we have chosen CR-39 detector that is an amorphous polymer consisting of short polyol chains joined by way of links containing diethylene glycol groups and carbonates into a dense three-dimensional network to a starting. In a track etch detector, a suitable chemical reagent preferentially attacks the damaged region to enlarge tracks. During the etching, the bulk material is attacked at a slower rate. The final geometry of the etched tracks is therefore determined in the simplest case by the simultaneous action of these two etching processes. The rate of these two etching processes is respectively designated as VT and VG. While the track etches rate VT depends on the specific ionization, both VG and VT are also dependent on the detector material, the etchant, its concentration and temperature of etching.

In figure 2, (a) Illustrates the shape of the etched track when $\mathrm{VG} / \mathrm{VT}$, (b) No track formation, as the surface was removed at a greater rate than the normal component of VT and (c) Sin VGNT is the critical angle Oc above which tracks are registered. In figure 3, initially, the track grows with its known cone-like structure, characterized by the two etch velocities VT and VG. However, because of increases in track etch rate near the end of the particle track, the track walls develop small curvature. When the track etching reaches the end of the particle range, further etching just extends the track at the bulk-etching rate and the track structure turns out to be progressively lost. Therefore, for a determination of the particle range from a measurement of the etched track length, it is necessary that etching time is just sufficient to fully etch the particle trajectory, but not too much beyond that. In addition, corrections for the track enlargements due to the bulk etching have to be done.

Energetic particles and polymer structure were colloids that leave a trail of broken chemical bonds within CR-39. The tracks could be visualized by etching the film in $\mathrm{NaOH}$ solution with standard etching conditions of $6 \mathrm{~N}, 600 \mathrm{C}$, and $6 \mathrm{Hrs}$ with constant stirring. At the point when immersed in a concentrated alkali solution and hydroxide ions attack and break the polymer structure, etching away most of the plastic at a nominally fixed rate. In CR39 films clamped on a clipping system, which is rotated by using a rotor system and is immersed in an etching solution. Heating control is maintained by using a microcontroller. A DC generator controls rotating speed and voltage.

After the chemical etching of a detector, an optical microscope can easily scan the tracks of a particle using ordinary magnification. Using ImageJ and python programmed for ellipse fitting, the average diameter and angle of the recoil of each tracks can be calculated. A calibration graph with proton energy and track diameter is used for calculating the recoil proton energy. From the calculated average diameter using a python programmed Ellipse.2, the recoil proton energy can be calculated using the equation $\left(0.0295956 * \mathrm{~d}^{\wedge} 2\right)-(1.16821 * \mathrm{~d})+12.674 \mathrm{~d}$, where $\mathrm{d}$ is the track diameter.

From the recoil proton energy, we can calculate the neutron energy by the equation 1 ,

$\mathrm{Ep}=\mathrm{En} \cos 2 \Theta$

Where Ep is the energy of the recoiled proton, En is the incident neutron energy and $\Theta$ is the angle of recoil respectively. Recoil angle $\theta$ is calculated from minor and major axes of the track ellipse. Numbers of scattering centers per unit area were calculated from the physical density and molecular composition. To get the spectral distribution of neutron, a histogram is plotted between this corrected counts and energy.The data analysis tools used are ImageJ, GNUPlot, Ellipse.2 and Exfor Data File. The microscope image of CR39 is analyzed with ImageJ software. From the image, a 2D profile of the area containing tracks was generated. Each track profiles 


\section{Analysis of Photo Neutron Dose to Patient from a 15 MV Linear Accelerator}

in this area were fitted with Ellipse equation using GNUPlot. In this study, the Python compiler is used for resolving neutron energy data from chemically etched CR-39 foil microscopic image, which is used to calculate the neutron dose.

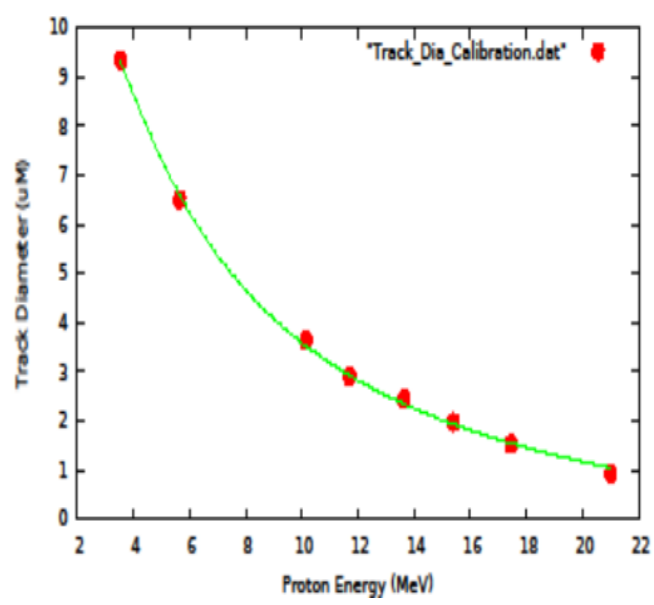

Fig.2 Calibration graph

Photonuclear reaction and therefore the cross-sections from the Geant 4 database permits to simulate neutron production approximately for all nuclei and all energies from the hadron's production threshold to about $40 \mathrm{TeV}$. These characteristics, along with the capability to model complex geometries make Geant4 particularly helpful for medical linear-accelerator simulation. Carrying out the simulations also Geant 4 was calculated due to the emitted photo neutrons of the patients, the most recent generation of old Geometry and Tracking. The phantom used in Geant 4 , of $10 \times 10 \times 30$ $\mathrm{cm} 3$, made of ICRU tissue including Carbon (11\%), Oxygen (76.2 \%), Hydrogen (10.1\%) and Nitrogen (2.6\%), which simulates a patient situated with its surface at $100 \mathrm{~cm}$ from the source. The figure 4 indicates schematically the phantom half, as used in the simulations. Dark grey cells positions were the neutron determinations are shown correspond to the axis and transver se positions are situated off an axis.

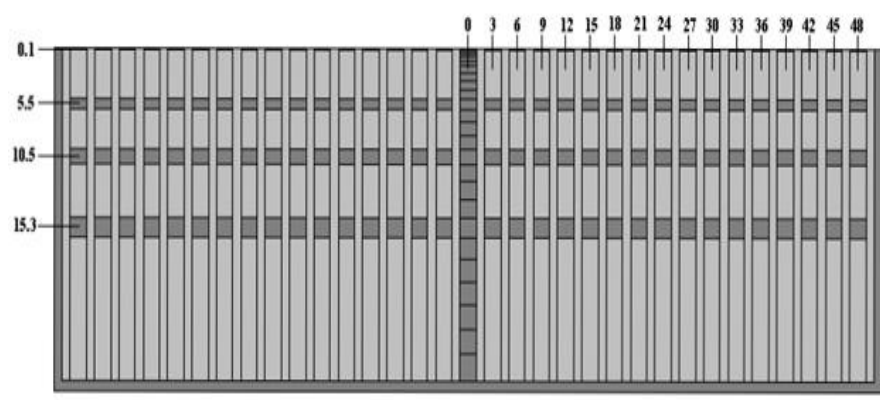

Fig.3 Dark grey cells positions in phantom half

In this work, Siemens Primus Plus linear accelerator which is in $15 \mathrm{MV}$ mode. The study was conducted on a patient with ca prostate treated with 3DCRT mode about a field size of
$15 \mathrm{~cm} \times 17 \mathrm{~cm}$. A patient is positioned on the top of the couch with an immobilization mask. A patient is aligned isocentrically. Three sets of CR39 films $(1 \mathrm{~cm} x 1 \mathrm{~cm}$ and density of $1.3 \mathrm{~g} / \mathrm{cm} 2$ ) one is placed on the mask at the center region of the field, one is placed on right side of the mask $5 \mathrm{~cm}$ lateral distance from the field and one is placed $5 \mathrm{~cm}$ inferiorly to the field. The patient planned with a box field technique with $15 \mathrm{MV} x$ rays and the rate of the dose $500 \mathrm{MU} / \mathrm{min}$. The AP field is delivered with the TPS calculated MU. The same repeated for with and without the patient. The films have taken for processing. The exposed film is taken out for etching. The exposed film also etched in $\mathrm{NaOH}$ solution with standard etching conditions of $6 \mathrm{~N}, 600 \mathrm{C}$, and $6 \mathrm{Hrs}$ with constant stirring.

An optical microscope is used to take an image of the etched film. Formed tracks are visible under the microscope. Using Image $\mathbf{J}$ software we can read out the tacks and using a python programmed for ellipse fitting to count the tracks. The data also binned with a width of $250 \mathrm{KeV}$ and intrinsic corrections applied by accounting elastic scattering cross section $(\sigma)$. Cross section data are collected from EXFOR-ENDF (Evaluated Nuclear Data File). The midpoint of each bin is taken as the average energy (E). A number of scattering centers per unit area calculated from the physical density and molecular composition. Counts in each bin were divided with the value of $(n \sigma)$ to get the real number of neutrons per energy bin, $(\phi(E))$ To get the spectral distribution of neutron, a histogram is plotted between this corrected counts and energy. A Geant4 application measures the neutron dose to the patient by the ICRU simulation phantom. Cross-section corrected counts and average energy are the input of the Geant 4 simulator.Geant 4 simulator calculates the dose due to each neutron with a given energy.

\section{RESULTS AND DISCUSSION}

Photo neutron spectrum and dose calculated. $15 \mathrm{MV}$ lead to photo neutron production from many of the elements including Tungsten, Lead, Tantalum Nitrogen, Phosphorus and so on. Particularly $207 \mathrm{~Pb}$ has photo neutron threshold as low as $6.74 \mathrm{MeV}$ and $182 \mathrm{~W}$ has threshold $8.04 \mathrm{MeV}$. Moreover, Nitrogen and Phosphorus have a lesser threshold for photo-proton emission at 7.55 $\mathrm{MeV}$ and 7.3 $\mathrm{MeV}$ respectively. Thus, the patient will be exposed to heterogeneous neutron energies from linac. This will add the contributions from patient supporting accessories. Further fast neutron may induce secondary neutron emission within the body. Neutrons produced in the tumor also will interact with peripheral tissues.

The energy of the recoiled protons has been estimated using track diameter - energy 
calibration for protons in standard etching conditions $(6 \mathrm{~N}$ $\mathrm{NaOH}, 600 \mathrm{C}, 6 \mathrm{Hrs}$ ) and the energy of incident neutrons was deducted as above. This process was repeated to each track containing the area. A profile of overlapping tracks analysed separately as follows. The track profile was first fitted with multiple Gaussian using a software program 'Candle' and the FWTM for each individual tracks were determined. Multiple ellipses were fitted with the equation for an individual constituent track by keeping previously evaluated FWTM as a reference and are analysed. In order to confirm that no characteristic's neutrons are missed from the observation in one field of view, Images for three fields of views are recorded in this way and summed over the views and the average is calculated for one field of view. However, in this percent observation, there is no characteristic missing is observed. The difference observed is well within the statistical fluctuation of less than $4 \%$.

The data also binned with a width of $250 \mathrm{KeV}$ and intrinsic corrections applied by accounting elastic scattering cross-section $(\sigma)$. Cross-section data collected from EXFOR-ENDF (Evaluated Nuclear Data File). The midpoint of each bin is taken as the average energy (E). A number of scattering centers per unit area have calculated from the physical density and molecular composition. Counts in each bin were divided with the value of $(n \sigma)$ to get the real number of neutrons per energy bin, $(\phi(E))$ To get the spectral distribution of neutron, a histogram is plotted between the corrected counts and energy as shown in figure 5.

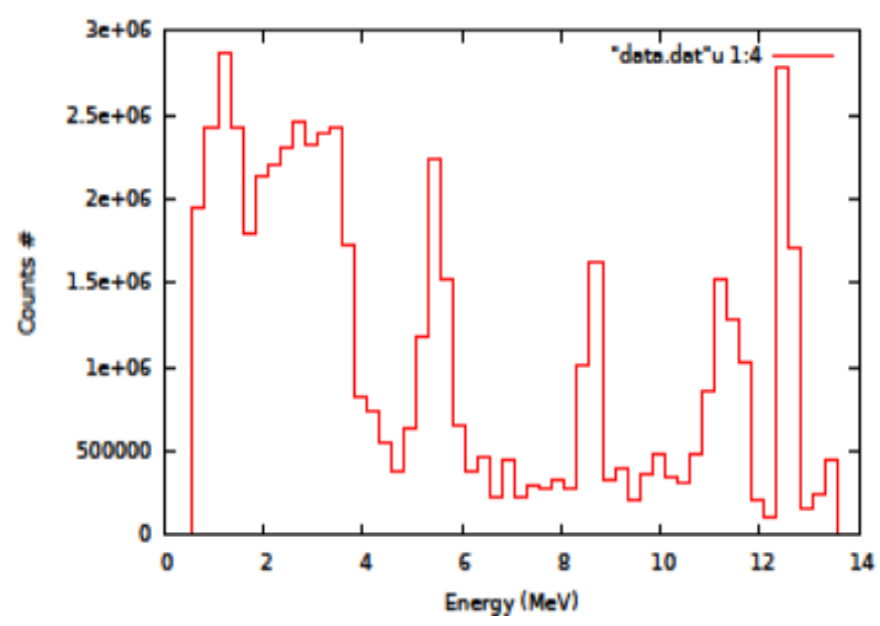

Fig.4 Histogram between the corrected counts and energy

The spectrum shows there are some of the significant amounts of high energy neutrons produced from the treatment head. The tracks due to scattered and secondary neutrons as well as low energy $(<0.7 \mathrm{MeV})$ are lost on etching. This leads to loss of information about the low energy neutrons produced. An alternative mechanism is to be employed for recording low energy primary neutrons. This is important as the typical radiation-weighting factor in this energy range is 20 .

However, contaminations due to secondary's and scattered neutrons could be very well eliminated thereby providing data on direct neutrons. Recoil energy of the residual nucleus is found to be less than $1 \mathrm{KeV}$ it will not affect the resolution of the characteristics of the neutron spectrum and hence can be neglected.

From the observed neutron peaks in the above spectra, the reaction channels were identified taking into account the various reactions having resonance reactions in the photon energy domain for elements of interest that are expected to be present in various components of treatment head. From the calculated neutron energy and counts, using Geant4 application, the neutron dose to the patient is measured.

\section{CONCLUSION}

The photo neutron spectrum and dose proceeding from a medical linear accelerator are calculated. 15 MV Medical Linear Accelerator shows a significant amount of neutrons produced from $(\Upsilon, \mathrm{n})$ reactions occurs at various parts of the machine. The consequence demonstrates which neutron dose equal from photo neutrons to patients in a conventional of the linac treatment ought not to be ignored even though it is less compared with the photon dose. The neutron dose equivalent, at the isocenter, also represents a risk for the healthy tissues and contributes to secondary malignancy insurgence. Neutron field assessment is in this way important to improve the treatment. The present technique, consisting of Geant4 simulation, can represent a reliable tool. The information likewise is used to assess the surrounding portion equal from photo neutrons to a patient accepting radiation treatment by a linear accelerator. Considering the radiation-weighting factor for neutrons, it is obvious that, the neutron dose considered in the treatment planning process, and we have to incorporate some mechanism in the radiotherapy treatment delivery for neutron free photons.

\section{REFERENCES}

1. Allen PD, Chaudhri MA. Photoneutron production in tissue during high energy bremsstrahlung radiotherapy. Physics in Medicine \& Biology. 1988 Sep;33(9):1017.

2. Cyriac TS, Musthafa MM, Raman RG, Haneefa KA, Bhasi S. Out-of-field photon dosimetry study between 3-D conformal and intensity modulated radiation therapy in the management of prostate cancer. International Journal of Radiation Research. 2015 $1 ; 13(2): 127-34$.

Apr

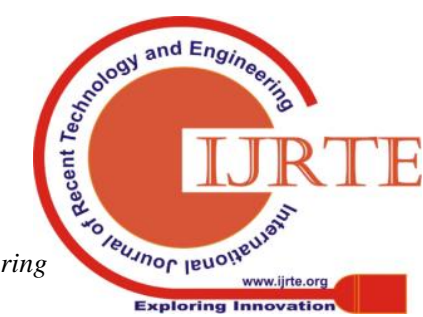


3. Kry SF, Salehpour M, Followill DS, Stovall M, Kuban DA, White RA, Rosen II. Out-of-field photon and neutron dose equivalents from step-and-shoot intensity-modulated radiation therapy. International Journal of Radiation Oncology* Biology* Physics. 2005 Jul 15;62(4):1204-16.

4. Kry SF, Salehpour M, Titt U, White RA, Stovall M, Followill D. Monte Carlo study shows no significant difference in second cancer risk between 6-and 18-MV intensity-modulated radiation therapy. Radiotherapy and Oncology. 2009 Apr $1 ; 91(1): 132-7$.

5. Rivard MJ, Coursey BM, DeWerd LA, Hanson WF, Saiful Huq M, Ibbott GS, Mitch MG, Nath R, Williamson JF. Update of AAPM Task Group No. 43 Report: A revised AAPM protocol for brachytherapy dose calculations. Medical physics. 2004 Mar;31(3):633-74

6. Niroomand-Rad A, Blackwell CR, Coursey BM, Gall KP, Galvin JM, McLaughlin WL, Meigooni AS, Nath R, Rodgers JE, Soares CG. Radiochromic film dosimetry: recommendations of AAPM radiation therapy committee task group 55. Medical physics. 1998 Nov;25(11):2093-115.

7. Barquero R, Edwards TM, Iñiguez MP, Vega-Carrillo HR. Monte Carlo simulation estimates of neutron doses to critical organs of a patient undergoing x-ray LINAC-based radiotherapy. Medical physics. 2005 Dec;32(12):3579-88.

\section{AUTHORS PROFILE}

Rajesh K.R, Research scholar, Department of Physics, Noorul Islam Centre for Higher Education, Kumaracoil, Kanya kumari District. Tamilnadu, India.

R. Ganapathi Raman, Associate Professor, Department of Physics, Noorul Islam Centre for Higher Education, Kumaracoil, Kanya kumari District, Tamilnadu, India. 